\section{CPT Coding Revisions}

The new laboratory CPT codes for each of the three major areas of clinical genetic laboratory testing is available in the following report. The availability of these new codes will greatly facilitate billing for existing and new tests and technologies used in laboratories. They also provide the basis for addressing reimbursement issues. Without codes that accurately describe the services currently provided, discussions of appropriate reimbursement are difficult. Cost analysis information about tests from your laboratory is welcomed. This will allow the College to provide HCFA and other payers with information to allow them to make an educated decision regarding appropriate rates of reimbursement. Please provide this information to the fax number given below.

In order to stay abreast of the rapid evolution of tests and technologies, we encourage you to communicate with the Committee on the Economics of Genetic Services about anticipated needs for CPT coding. It can take from 6 months to years to fully develop the information and proposals for CPT modifications or additions.

Direct all comments and inquiries to the attention of "CPT Coding Revisions" and send via fax to 301-571-1895.

\section{NEW LABORATORY CPT CODES}

Major revisions to the CPT coding of genetic testing have been approved by the AMA CPT Editorial Panel and go into effect on January 1, 1999. The following will provide you with the revised CPT codes (new, modified, and deleted) and offer an overview of how they might be combined or "stacked" to describe particular tests. There is also a brief discussion of important considerations in incorporating these into your laboratory's or institution's billing and reimbursement systems.

As a point of reference to facilitate reviewing the individual lists of codes, note the following. The codes are presented in two columns. The first column shows the codes' 1998 status and language, while the second column shows this for 1999. Where a code did not exist in 1998, we have noted that in the $1998 \mathrm{col}-$ umn and inserted the language of the new code in the $1999 \mathrm{col}-$ umn. Deleted codes or modified codes have a line through the language being removed. When modified, the new language is underlined in the 1999 column. When a code description is indented, the first part of the code description is determined by the language of the code immediately preceding it. If that code is also indented, continue back through the codes to the first one which is not indented. The language preceding the semicolon applies to each of the indented codes following it. Where the code has not changed, "same" is noted in the 1999 column. The codes for each of the areas of genetic testing are appended.

\section{Clinical Cytogenetics}

Overview: There are several significant changes within the Cytogenetics codes. In general terms, the codes have been refocused to distinguish between testing for cancer versus constitutional abnormalities. The major changes include 1 ) the addition of CPT codes to describe FISH tests; 2) a new code for cancer cytogenetic analyses; 3 ) a new pair of codes to describe the freezing of viable cell lines and their subsequent thawing and reestablishment in cell culture to be used for tests (usually biochemical) requiring viable cells which are commonly sent to reference laboratories. These codes will likely be used by cytogenetics laboratories or biochemical genetics laboratories. 4) There is also a new $\mathrm{CPT}$ code for the reporting and interpretation of results of cytogenetic and molecular cytogenetic testing which is generally used when results and interpretation are abnormal and/or complex.

The CPT Codes: See Appendix 1 for CPT codes 88230-88299.

Example: A patient presents with an interrupted aortic arch and hypocalcemia. Your laboratory is asked to consider a diagnosis of DiGeorge syndrome by performing a complete chromosome analysis and fluorescence in situ hybridization (FISH) testing for a microdeletion in $22 \mathrm{q} 11.2$ from a blood sample.

The CPT codes that describe this test are:

88230 lymphocyte cell culture

88262 chromosome analysis; count 15-20 cells, 2 karyotypes, with banding

USE IF CHROMOSOME ANALYSIS IS INCLUDED IN STUDY

88271 molecular cytogenetics; DNA probe, each USE ONCE FOR EACH PROBE USED

88271 see above SECOND PROBE, IF USED

88273 molecular cytogenetics; chromosomal in situ hybridization, analyze $1-30$ cells

88291 cytogenetic and molecular cytogenetics, interpretation and report

IF RESULT REQUIRES COMPLEX INTERPRETATION

\section{Molecular Diagnostics}

Overview: The primary changes to this area involve generalizing language to cover nucleic acids rather than DNA and the addition of several codes reflecting more recent technologies used in molecular diagnosis. Note that, for the most part, the codes discussed here have been developed to apply to the analysis of the human genome since a significant number of new codes exist for the molecular identification of infectious agents (see 87470-87799). However, the availability of codes for specific infectious agents does not necessarily preclude the use of codes within this area.

The CPT Codes: See Appendix 2 for CPT codes 83890-83912.

Example: A newborn presents with meconium ileus. From a blood sample, test for mutations in the CFTR gene associated with cystic fibrosis. Your laboratory does this test using DNA sequencing. You test for five mutations that lie within five independent stretches of DNA that are individually amplified by PCR.

The CPT codes that describe this test are:

83890 molecular isolation or extraction

83901 amplification of patient nucleic acid, (e.g., PCR), single primer pair, each primer pair USE THIS CODE 5 TIMES FOR EACH PRIMER PAIR 
3904

\section{USED TO AMPLIFY}

mutation identification by sequencing, single segment, each segment

USE THIS CODE 5 TIMES FOR EACH SEGMENT SEQUENCED

3912 interpretation and reporting

\section{liochemical Genetics}

Overview: The goal for reworking the CPT coding for Biohemical Genetics was to move away from listing individual amino cids or organic acids, some of which have CPT codes already, to I more general approach. Furthermore, we wanted to provide for fualitative and quantitative assays, for single analytes, multiple inalytes, and profiles, and to have the newer technologies more :ommon to our field included. A few new analytes common to jiochemical genetic testing have also been added. Note that new and modified codes are available in the cytogenetics section for zell culture. The language has been changed such that cell culture sn't specifically for the purpose of cytogenetic testing but, rather, is for testing for either nonneoplastic or neoplastic disorders. Furthermore, codes for cryopreservation as well as the thawing and reestablishment of cell lines, often needed for sequential distribution of cells to reference laboratories, are available.

Lastly, a code for interpretation and reporting was proposed but denied. The reasoning was that this activity is commonly a professional service component that is performed in the course of a consultation and, therefore, billable under that group of services.

The CPT Editorial Panel recently decided to incorporate all of our new codes into the chemistry section rather than have a subsection on Biochemical Genetics in order to accommodate others using these codes.

The CPT Codes: See Appendix 3 for the list of CPT codes in the Biochemical Genetics area. Note that this list is not all-inclusive due to the number of analytes in the existing CPT code book. Rather, the lists reflect codes that are new or modified only and applicable to this area of testing. For those codes for which the description of the code states "analyte not specified elsewhere," the existing analyte code description likely includes the analytical technique historically used for that test. You would stack the new codes in instances in which you apply a new analytical code. Lastly, the term "specimen" has been used to allow for the testing of multiple tissues in situations in which the ratio between tissues is important or multiple tissue assessments are clinically relevant.

Example: Your laboratory is asked to analyze a urine specimen for acylcarnitines. The test done in your laboratory involves profiling the carnitine esters using fast atom bombardment and electrospray ionization coupled with tandem mass spectrometry.

The CPT codes that describe this test are:

82016 acylcarnitines; qualitative, each specimen

83788 mass spectrometry and tandem mass spectrometry

\section{General}

Important considerations: The availability of particular CPT codes does not necessarily guarantee their reimbursement. In most situations, diagnostic coding (ICD) must support the use of the test in any particular situation. Also, note that no relative value units (RVUs) have been assigned to the base code for reporting and interpretation of complex cytogenetic results. RVUs are assigned to professional services while the reimbursement for analytical codes is assigned by the Health Care Financing Administration (HCFA). In general terms, professional services are considered those reimbursable to an individual with a UPN, a number which identifies a physician within the Medicare system. Hence, only physicians can be reimbursed for the use of this code when Medicare is billed. This does not necessarily preclude their use being built into contracts with referral bases. We are beginning to pursue acquiring restricted licenses for Board-certified $\mathrm{PhD}$ lab directors, which would allow them to bill for professional services within their area of certification. This would parallel the actions in a few states in which this privilege already exists.

\section{DISCLAIMERS IN REPORTS OF GENETIC TESTS USING ANALYTE SPECIFIC REAGENTS}

Pursuant to a new FDA rule, effective November 23, 1998, laboratories reporting out results of tests using analyte specific reagents (ASRs), including molecular probes, that have not been approved by the FDA must include a specific disclaimer on the test report. ASRs are reagents composed of chemicals or antibodies that constitute the "active" ingredients of an in-house developed test. Manufacturers are required to label all such products as ASRs. The required disclaimer states as follows:

"This test was developed and its performance determined by (laboratory name). It has not been cleared or approved by the U.S. Food and Drug Administration."

Because this statement may cause confusion among physicians, patients, and payers regarding whether such tests are clinically necessary and reimbursable, laboratories may wish to add clarifying language after the disclaimer, such as the following:

"The FDA has determined that such clearance or approval is not necessary. This test is used for clinical purposes."

Laboratories also may wish to add the following:

"Pursuant to the requirements of CLIA ' 88 , this laboratory has established and verified the test's accuracy and precision."

Any clarifying language must be in addition to the exact language required in the FDA disclaimer.

Clinical laboratories using ASRs must verify or establish, for each use of an ASR, the performance characteristics, e.g., accuracy, precision, analytical sensitivity and specificity, etc. 42 CFR $\$ 493.1213$.

Geneticists who provide interpretations of tests performed using ASRs, and laboratories that report out results of tests performed by other laboratories, also must include the FDA-required disclaimer on test reports. Although the disclaimer requirement does not apply to ASRs developed and used exclusively in-house (i.e., home-brew ASRs), which are not currently regulated by the FDA, some variation of the required disclaimer and clarifying language is recommended. The disclaimer also does not apply to ASRs that are sold and used as components of tests or kits (in vitro diagnostics) that have been cleared or approved by the FDA. Finally, the disclaimer is not required for tests using reagents that are labeled for "Research Use Only" (RUOs) or for "Investigational Use Only" (IUOs). Such reagents are subject to FDA approval but have not been approved for clinical use. 


\section{Appendix 1}

Clinical Cytogenetics CPT Codes

\begin{tabular}{|c|c|c|c|}
\hline \multicolumn{2}{|c|}{1998 CPT Code $(s)$} & \multicolumn{2}{|c|}{1999 CPT Code(s) } \\
\hline 88230 & tissue culture for chromosome analysis; lymphocytes & 88230 & $\begin{array}{l}\text { tissue culture for non-neoplastic disorders; } \\
\text { lymphocyte }\end{array}$ \\
\hline 88233 & skin or other solid tissue biopsy & 88233 & same; but now for non-neoplastic \\
\hline 88235 & amniotic fluid or chorionic villus cells & 88235 & same; but now for non-neoplastic \\
\hline 88237 & $\begin{array}{l}\text { tissue culture for chromosome analysis; bone marrow } \\
\text { (myeloid) cells }\end{array}$ & 88237 & $\begin{array}{l}\text { tissue culture for neoplastic disorders; bone marrow (mycloid), } \\
\text { blood cells }\end{array}$ \\
\hline \multirow[t]{3}{*}{88239} & other tissue & 88239 & other tissue solid tumor \\
\hline & None; new code & 88240 & cryopreservation, freezing and storage of cells, each cell line \\
\hline & None; new code & 88241 & thawing and expansion of frozen cells, each aliquot \\
\hline 88245 & $\begin{array}{l}\text { chromosome analysis for breakage syndromes; score } 25 \text { cells } \\
\text { (SCE study), count } 5 \text { cells, } 1 \text { karyotype, with banding }\end{array}$ & 88245 & $\begin{array}{l}\text { chromosome analysis for breakage syndromes; } \\
\text { (SCE), 20-25 cells }\end{array}$ \\
\hline \multirow[t]{2}{*}{88248} & $\begin{array}{l}\text { score } 100 \text { cells, count } 20 \text { cells, } 2 \text { karyotypes, with banding } \\
\text { (e.g., ataxia telangiectasia, Fanconi anemia) }\end{array}$ & 88248 & $\begin{array}{l}\text { baseline breakage, score } 50 \text { - } 100 \text { cells, count } 20 \text { cells, } 2 \text { karyotypes, } \\
\text { (e.g., for ataxia telangiectasia, Fanconi anemia, } \\
\text { fragile X) }\end{array}$ \\
\hline & None; new code & 88249 & $\begin{array}{l}\text { score } 100 \text { cells, clastogen stress, (e.g., diepoxybutane, mitomycin } \\
\mathrm{C} \text {, ionizing radiation, UV radiation) }\end{array}$ \\
\hline 88250 & $\begin{array}{l}\text { chromosome analysis for fragile X linked mental retardation, } \\
\text { score } 100 \text { cells, count } 20 \text { cells, } 2 \text { karyotypes with banding }\end{array}$ & 88250 & $\begin{array}{l}\text { Code Deleted-See } 88248 \\
\text { C, }\end{array}$ \\
\hline 88260 & chromosome analysis, count 5 cells, screening with banding & 88260 & 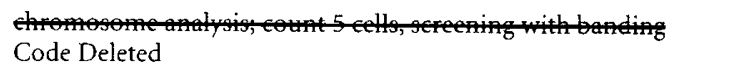 \\
\hline 88261 & count 5 cells, 1 karyotype, with banding & 88261 & $\underline{\text { chromosome analysis, }}$ count 5 cells, 1 karyotype, with banding \\
\hline 88262 & count $15-20$ cells, 2 karyotypes, with banding & 88262 & Same \\
\hline 88263 & count 45 cells for mosaicism, 2 karyotypes, with banding & 88263 & Same \\
\hline 88264 & None; new code & 88264 & analyze $20-25$ cells \\
\hline 88267 & $\begin{array}{l}\text { chromosome analysis, amniotic fluid or chorionic villus, } \\
\text { count } 15 \text { cells, } 1 \text { karyotype, with banding }\end{array}$ & 88267 & Same \\
\hline \multirow[t]{6}{*}{88269} & $\begin{array}{l}\text { chromosome analysis, in situ for amniotic fluid or chorionic } \\
\text { villus, count } 15 \text { cells, } 1 \text { karyotype, with banding }\end{array}$ & 88269 & Same \\
\hline & None; new code & 88271 & $\begin{array}{l}\text { molecular cytogenetics; DNA probe, each (e.g., FISH) } \\
\text { None; new code }\end{array}$ \\
\hline & None; new code & 88272 & $\begin{array}{l}\text { chromosomal in situ hybridization, analyze } 3-5 \text { cells, } \\
\text { (e.g., for derivatives or markers) }\end{array}$ \\
\hline & None; new code & 88273 & $\begin{array}{l}\text { chromosomal in situ hybridization, analyze } 10-30 \text { cells (e.g., for } \\
\text { microdeletions) }\end{array}$ \\
\hline & None; new code & 88274 & interphase in situ hybridization, analyze $25-99$ cells \\
\hline & None; new code & 88275 & interphase in situ hybridization, analyze $100-300$ cells \\
\hline 88280 & chromosome analysis; additional karyotypes, each analysis & 88280 & Same \\
\hline 88283 & additional specialized banding technique (e.g., NOR C-banding) & 88283 & Same \\
\hline 88285 & additional cells counted, each study & 88285 & Same \\
\hline 88289 & additional high resolution study & 88289 & Same \\
\hline 38291 & None; new code & 88291 & $\begin{array}{l}\text { cytogenetics and molecular cytogenetics, interpretation and } \\
\text { reporting }\end{array}$ \\
\hline 38299 & unlisted cytogenetics study & 88299 & Same \\
\hline
\end{tabular}

Crossed-out text has been deleted from 1999 codes, and underlined text has been added to 1999 codes. 
Appendix 2

Molecular Diagnostics CPT Codes

\begin{tabular}{|c|c|c|c|}
\hline \multicolumn{2}{|c|}{1998 CPT Code(s) } & \multicolumn{2}{|c|}{1999 CPT Code(s) } \\
\hline 83890 & molecular diagnostics; molecular isolation or extraction & 83890 & molecular diagnostics; molecular isolation or extraction \\
\hline & None; new code & 83891 & isolation or extraction of highly purified nucleic acid \\
\hline \multirow[t]{2}{*}{83892} & enzymatic digestion & 83892 & enzymatic digestion \\
\hline & None; new code & 83893 & dot/slot blot production \\
\hline 83894 & separation (e.g., dot blot, electrophoresis) & 83894 & $\begin{array}{l}\text { separation by gel electrophoresis (e.g. } \\
\text { agarose, polyacrylamide) }\end{array}$ \\
\hline \multirow[t]{2}{*}{83896} & nucleic acid probe, each & 83896 & Same \\
\hline & None; new code & 83897 & nucleic acid transfer (e.g., Southern, Northern) \\
\hline \multirow[t]{2}{*}{83898} & $\begin{array}{l}\text { molecular diagnostics; amplification, e.g., polymerase chain } \\
\text { reaction (PCR) }\end{array}$ & 83898 & $\begin{array}{l}\text {; amplification, e.g., polymerase chain } \\
\text { reaction, each of patient nucleic acid (e.g., PCR, LCR, RT-PCR), } \\
\text { single primer pair, each primer pair }\end{array}$ \\
\hline & None; new code & 83901 & $\begin{array}{l}\text { amplification of patient nucleic acid, multiplex, each multiplex } \\
\text { reaction }\end{array}$ \\
\hline \multirow[t]{5}{*}{83902} & reverse transcription & 83902 & Same \\
\hline & None; new code & 83903 & $\begin{array}{l}\text { mutation scanning, by physical properties (e.g., single strand } \\
\text { conformational polymorphisms (SSCP), heteroduplex, denaturing } \\
\text { gradient gel electrophoresis (DGGE), RNA'ase A), single } \\
\text { segment, each }\end{array}$ \\
\hline & None; new code & 83904 & $\begin{array}{l}\text { mutation identification by sequencing, single segment, } \\
\text { each segment }\end{array}$ \\
\hline & None; new code & 83905 & $\begin{array}{l}\text { mutation identification by allele specific transcription, single } \\
\text { segment, each segment }\end{array}$ \\
\hline & None; new code & 83906 & $\begin{array}{l}\text { mutation identification by allele specific translation, single segment, } \\
\text { each segment }\end{array}$ \\
\hline 83912 & interpretation and reporting & 83912 & Same \\
\hline
\end{tabular}

Crossed-out text has been deleted from 1999 codes, and underlined text has been added to 1999 codes. 
Appendix 3

Clinical Biochemical Genetics CPT Codes

\begin{tabular}{|c|c|c|c|}
\hline \multicolumn{2}{|c|}{$1998 \mathrm{CPT}$ Code(s) } & \multicolumn{2}{|c|}{1999 CPT Code(s) } \\
\hline & None; new code & 82016 & acylcarnitines, qualitative, each specimen \\
\hline & None; new code & 82017 & quantitative, each specimen \\
\hline & None; new code & 82127 & amino acids; single, qualitative, each specimen \\
\hline 82128 & amino acids; qualitative & 82128 & multiple, qualitative, each specimen \\
\hline 82130 & amino acids; urine or plasma, chromatographic fractionations & 82130 & Deleted-see $82131,82136,82139$ \\
\hline \multirow[t]{5}{*}{82131} & amino acids, quantitation, each & 82131 & single qualitative, each specimen \\
\hline & None; new code & 82136 & amino acids, 2 to 5 amino acids, quantitative, each specimen \\
\hline & None; new code & 82139 & amino acids, 6 or more amino acids, quantitative, each specimen \\
\hline & None; new code & 82261 & biotinidase, each specimen \\
\hline & None; new code & 82379 & carnitine (total and free), quantitative, each specimen \\
\hline 82486 & $\begin{array}{l}\text { chromatography, qualitative; column (e.g., gas liquid or high } \\
\text { performance liquid chromatography), analyte not elsewhere } \\
\text { specified }\end{array}$ & 82486 & $\begin{array}{l}\text { chromatography, qualitative; column (e.g., gas liquid or HPLC } \\
\text { specified }\end{array}$ \\
\hline \multirow[t]{18}{*}{82491} & $\begin{array}{l}\text { chromatography, quantitative, column (e.g.. gas liquid or high } \\
\text { performance, liquid chromatography) analyte not elsewhere } \\
\text { specified, single stationary and mobile phase }\end{array}$ & 82491 & $\begin{array}{l}\text { chromatography, quantitative, column (e.g., gas liquid or } \\
\text { not elsewhere specified, single stationary and mobile phase }\end{array}$ \\
\hline & None; new code & 82492 & multiple analytes, single stationary and mobile phase \\
\hline & None; new code & 82541 & $\begin{array}{l}\text { column chromatography/mass spectrometry (e.g., GC/MS, or } \\
\text { HPLC/MS), analyte not elsewhere specified, single stationary and } \\
\text { mobile phase }\end{array}$ \\
\hline & None; new code & 82542 & quantitative, single stationary and mobile phase \\
\hline & None; new code & 82543 & $\begin{array}{l}\text { stable isotope dilution, single analyte, quantitative, single } \\
\text { stationary and mobile phase }\end{array}$ \\
\hline & None; new code & 82544 & $\begin{array}{l}\text { stable isotope dilution, multiple analytes, quantitative, single } \\
\text { stationary and mobile phase }\end{array}$ \\
\hline & None; new code & 82657 & $\begin{array}{l}\text { enzyme activity in blood cells, cultured cells, or tissue, not elsewhere } \\
\text { specified; nonradioactive substrate, each specimen }\end{array}$ \\
\hline & None; new code & 82658 & radioactive substrate, each specimen \\
\hline & None; new code & 82726 & very long chain fatty acids \\
\hline & None; new code & 83070 & beta-hexosaminidase, each assay \\
\hline & None; new code & 83788 & $\begin{array}{l}\text { mass spectrometry and tandem mass spectrometry (MS, MS/MS), } \\
\text { analyte not elsewhere specified; qualitative, each specimen }\end{array}$ \\
\hline & None; new code & 83789 & quantitative, each specimen \\
\hline & None; new code & 83918 & organic acids; quantitative, each specimen \\
\hline & None; new code & 83919 & qualitative, each specimen \\
\hline & None; new code & 84376 & $\begin{array}{l}\text { sugars (mono-, di, and oligosaccharides); single qualitative, } \\
\text { each specimen }\end{array}$ \\
\hline & None; new code & 84377 & multiple qualitative, each specimen \\
\hline & None; new code & 84378 & single quantitative, each specimen \\
\hline & None; new code & 84379 & multiple quantitative, each specimen \\
\hline
\end{tabular}

Crossed-out text has been deleted from 1999 CPT codes, and underlined text has been added to 1999 codes. 